Andrew Wilson

Lancaster University

Małgorzata Haładewicz-Grzelak

Politechnika Opolska

\title{
Sports Utility Semiotics: A semantic differential study of symbolic potential in
} automobile design

\section{Summary}

The article aims to illuminate the issue of symbolic potential in post-modern society through a semiotic study of car design. In Baudrillard's terminology, we explore the experience and sociological and psychological materiality of objects which, being above objects' perceptible materiality, modify constantly the integrity of technological systems (Baudrillard 2005[1968]: 6). The target concepts are analyzed through Baurdillard's lens of symbolic capital and his technological system of objects, coupled with a method of semantic differentiality (SD; e.g. Osgood 1976, 1981) against the insights of Tartu semiotics. Such a complex framework helps to establish affective attitudes of the subjects towards scales selected for their perceptual saliency. The analysis is based on the responses of students in a Polish university who were administered an instrument comprising 14 concepts and 37 scales. The results of statistical analysis yield a semantic space with two factors: potency and activity/dynamism, which we shall call social prestige. At this stage of the analysis we could not determine the evaluation factor. The scales that loaded significantly showed that there is indeed an increment of perceptual saliency in both extracted factors in the case of target stimuli (pickups and SUVs).

Key words: semantic differential; luxury; semiotics of economics; Roland Barthes; Jean Baudrillard; symbolic prestige; car design.

\section{Preliminaries}

C.E. Osgood, G.J. Suci and P.H. Tannenbaum have shown that by posing subjects a series of questions about a specific concept as seven-point scales, with opposing adjectives at each end, we are able to sift out general patterns from them using statistical techniques (Danesi 2009[1999]: 27). As Danesi further observes, a semantic differential is thus a technique for "fleshing out the connotation of words" or constructing "connotative profiles" Danesi 
(2008[1999]: 27). Crucially, "research utilizing the semantic differential has shown that, although the connotations of specific concepts are subject to personal interpretation and subjective perceptions, the range of variation is rarely random or haphazard. In other words, the experiments using the semantic differential have shown that connotation is constrained by culture" (Danesi 2008[1999] : 27).

This research aims to put to use such a technique of 'connotative profiles' in a semiotic study of symbolic potential in car design. We use a meta-theory of Baudrillard's work on the technological system of objects, coupled with Tartuvian semiotics, to set the background for semantic differential exploration of the tests applied to undergraduate students in a Polish university. The work is structured as follows. The first section addresses some implications of the interrelation between semiotics and economics by considering the work of Roman Jakobson, Roland Bathes and Mortleman's research on the concept of luxury. The reason for this overview is to bring to light the contractual aspect of semiotics. The next section briefly reviews semiotic work on automobiles. The conclusion drawn is that, in general, the researcher's voice is mainly heard in this type of study and the researcher's personal semiotic interpretation is given, hence the need for a discussion as conducted in the present paper; namely, this is an attempt to elaborate on the 'rough data' collected through questioning the subjects. The fourth section presents Osgood's method of semiotic differential as a viable analytic tool and the fifth takes a look at car design through the lens of the dichotomy of 'vernacular' and 'skeoumorphic', with particular emphasis on the increase in semiotic potential in subsequent vehicle models. The subsequent sections describe the analytical procedure and the research instrument, followed by a discussion and a conclusion.

\section{A semiotic balance sheet of economic success and failure}

L'étude des signes ne peut cependant se limiter à de tels systèmes uniquement sémiotiques, mais doit également prendre en considération des structures sémiotiques appliqués, comme l'architecture, le vêtement, ou la cuisine" (Jakobson 1973: 98).

Jakobson (1973: 36) points out that during the secular history of economy and linguistics, on several occasions the two disciplines came close to each other. The rapprochement was, so to speak, from both sides. The scholar mentions such names as Turgot or Adam Smith as economists who dealt with linguistics. In particular, "l'influence de G. Tarde sur la doctrine de Saussure en matière de circuit, d'échange, de valeurs, d'entrée et de la sortie, de producteur et de consommateur est bien connue" (Jakobson 1973: 36). What is more, the fundamental 
economic concepts were quite often the object of provisory semiotic interpretations. For example, citing Feruccio Rossi-Landi, Jakobson assumes that

l'économie au sens propre est l'étude du secteur de la communication non verbal qui consiste dans la circulation d'un type particulier de messages habituellement appelés 'marchandises' ; pour employer une formule plus brève : l'économie est l'étude des messages-marchandises' (235, p.62). Pour éviter une extension métaphorique du terme 'language', il est peut être préférable de considerer la monnaie comme une système sémiotique à destination particulière. Si l'on veut étudier avec exactitude ce moyen de communication, il faut soumettre les processus et les concepts en jeu à une interpretation sémiotique (...). En realité, 'aspect symbolique, verbal, des transactions économiques mérite une étude interdisciplinaire systématique qui devrait être l'une des tâches les plus fructueuses de la sémiotique appliquée" (Jakobson 1973: 36).

Jakobson also says that that integrated science of communication comprises not only semiotics per se, i.e. the study of messages as such and the codes on which they rely, but also the disciplines in which messages play a pertinent but secondary role. The scholar agrees that semiotics occupies a central position in the general science of communication where it underlies other branches, while it itself encompasses linguistics, which in turn, being at the centre of semiotics, underpins all other sectors. Furthermore, Jakobson points out that three sciences, constituting an ensemble, encompass one another and represent three degrees of increasing generalization: 1) the study of the communication of verbal messages (linguistics); 2) the study of communication in any message - semiotics (comprising the communication of verbal messages); 3) the study of communication or social and economic anthropology (comprising the communication of messages) (cf. Jakobson 1973: 37).

Applied work on the semiotics of sign systems was also undertaken by Barthes (1986). Barthes' view contradicts the Jakobsonian and traditional Saussurean position of the interrelation between language and semiotics. Barthes, in contrast to Jakobson, stresses the priority of language in semiological processing: he agrees that objects, images or patterns can signify, but never autonomously. It means that every semiological system has a linguistic admixture: "[a]s for the collection of objects (clothes, food), they enjoy the status of systems only insofar they pass through the rally of language, which extracts their signifiers (in the form of nomenclature) and names their signifieds (in the form of usages or reasons)" (Barthes 1986: 10). He further points out that it seems impossible to claim the existence of a system of images or objects whose signifieds exist independently of language: "to perceive what a substance signifies is inevitably to fall back on the individuation of language" (Barthes 1986: 10). As such, it is semiology which is, for Barthes, part of linguistics, in particular the part covering large signifying units of discourse. Using a dialectic (the contradiction of two conflicting forces, which are seen as determining factors in their continuing interaction) of 
'language' and 'speech' in their, so to speak, transcendental aspect, as a general category, Barthes proceeds to the analysis of signifying systems such as, e.g., the garment system or the food system.

Another area of pertinence to the present work is Barthes's emphasis on the value in linguistics and semiology. Following Saussure, Barthes points out that economics and linguistics share several similarities: in both realms we are dealing with a system [our emphasis] of equivalence between two different things (e.g. work and reward, signifier and signified). Notwithstanding, in either case, this equivalence is not isolated, because if we alter one of its terms then, concomitantly, the whole system changes by degrees. There are thus two prerequisites for a sign (or economic value) to exist: a possibility on the one hand to exchange dissimilar things and, on the other, to compare similar things. Citing Saussure, Barthes observes that value seems more important than signification: "What quantity of idea or phonic matter a sign contains is of less import than what it is around it in the other signs" (Barthes 1986: 55). From this particular position onwards, the importance of the context follows, hence Barthes' stand on neutralization, understood as a pressure of the syntagm on the system, "and we know that the syntagm, which is close to speech, is to a certain extent a factor of defaulting; the strongest systems (like the Highway Code) have poor syntagms, the great syntagmatic complexes (like the image system) tend to make meaning ambiguous" (Barthes 1986: 85f).

For Barthes, signification is a process, the act of binding the signifier to the signified. In this understanding, the two are, at the same time, terms and in a relation. The association of sound and representation in language is not exactly arbitrary, as Barthes points out, for no individual is free to modify it - so it can be called unmotivated (Barthes: 1986: 48). Barthes concludes that, in language, "the link between signifier and signified is contractual in its principle, but that this contract is collective, inscribed in a long temporality (...) and, consequently it is, as it were, naturalized" (Barthes 1986: 51). What follows, for Barthes, is that "a system is arbitrary when its signs are founded not by convention, but by unilateral decision: the sign is not arbitrary in language but it is in fashion; and we shall say that a sign is motivated when the relation between its signifier and signified is analogical" (Barthes 1986: $51)$.

This contractual aspect of some semiotic systems is most conspicuous in the concept of luxury. Mortelman (2005) provides a retrospective of the topic, starting from Plato, and points out that, historically, there have been two approaches to luxury: the negative one, which blamed luxury either for moral inappropriateness (in Ancient Greece) and/or for 
eroding the strength of society (during the Renaissance). The positive approach (e.g. in the works of D'Avanel) stressed the fact that, sooner or later, ordinary people can finally reap the rewards of industrialization (Mortelmans 2005: 500). The French Revolution seemed to put an end to a link between position, power and luxury. "Luxury became more than ever a consumer product being sold at the market to those who can afford it. To put it in the language of Talcott Parsons: luxury loses its ascribed role and gets an achieved role" (Mortelmans 2005: 502). The author further discerns another divide in the study of luxury, basing it on the approach to the need-wants distinction: naturalist and idealist. Mortelmans concludes his review by stating that it is impossible to define luxury in an absolute way: it might be present in all cultures at all times. Crucially, any product can be turned into a luxury product as soon as certain conditions are met. It is vital to take this cumulative aspect into account as there have to be several characteristics that occur simultaneously. This is what leads to a narrow definition of luxury as encompassing "scarce products with an objective or symbolic extra value, with a higher standard of quality, and with a higher price than comparable products" (Mortelmans 2005: 507). However, a fuller understanding is possible when taking into account semiotic criteria, such as sign value. Mortelmans posits that "[t]he sign-value of an object is a catch-all in which several diverse significations (beyond usevalue, exchange value, and symbolic value) can be brought together". Sign-value accentuates the polysemic character of material culture without fixing its actual meaning. A broader definition of luxury thus sees "luxury products as those products that have a sign value on top of (or in substitution of) their functional or economical meaning" (Mortelmans 2005: 510). It is the semantic space of sign value thus understood which we set off to investigate in the present paper.

Of importance for our analytical paradigm is also the work of Jean Baudrillard on the technological systems of objects. As with every prominent scholar, Baudrillard's thoughts evolved through the years. ${ }^{1}$ We will rely in particular on his yearly books, in which Baudrillard studies the influence of technology on society: The System of Objects and Consumer society. Baudrillard posits there that objects have become signs and that their value is determined by a cultural code. In particular, consumer objects are seen as a classification system, coding the behaviour of social actors. What actually follows from such an assumption is that consumer objects should be analyzed by using linguistic, rather than social categories:

\footnotetext{
${ }^{1}$ See Genosko (1994) on Baudrillard's 'tempestuous' encounters with semiotics, evolving around Baurdillard's battle cry that 'les signes doivent brûler'.
} 
Technology gives us a rigorous account of objects in which functional antagonisms are dialectically resolved into larger structures. Every transition from a system to another, better integrated system, every commutation within an already structured system (...), precipitates the emergence of meaning, an objective pertinence that is independent of individuals who are destined to put it into preparation; we are in effect at the level of language here, and, by analogy with linguistic phenomena, those simple technical elements -different from real objects upon whose interplay technological revolution is founded - might well be dubbed 'technemes' (Baudrillard: 2005[1968]: 5).

The thematic focus of Baudrillard in these works is how an individual experiences technology in everyday life. The priority is put on forms which, freed from both practical functions and the human gestural system, become relative with regard to one another and to space, to which they provide 'rhythm'. Thus, "it is only the form which is present - which wraps that mechanism in its perfection and confines it within its contours" (Baudrillard 2005[1968]: 56).

Baudrillard's stand on luxury can be also superscripted by systemic and structural relations and cast in terms of a dyad: affluence and waste. For Baudrillard, the sign of affluence is not defined in neutral terms, as merely a 'sufficient' amount: enough is not enough. What gives a sign of opulence the required prominence is the fact of being superfluous, of going beyond the level of utility. To that end, affluence needs the support of 'waste': "[i]t is that wastage which defies scarcity and, contradictorily, signifies abundance. It is not utility, but that wastage which, in its essence, lays down the psychological, sociological and economic guidelines for affluence" (Baudrillard 1998: 45). In this work we aim to investigate precisely a semiotic dimension of the superfluous.

\section{Automotive semiotics}

In order to become object of consumption, the object must become sign. That is to say: it must become external, in a sense, to relation it signifies (Baudrillard 2005: 218).

As Mick et al. observe, automobiles, as culturally intensive products, have been a common topic for semiotic analysis, "with their meanings often tied to Western science and technology, sociocultural status and power, and personal freedom and escape" (Mick et al. 2002: 46). The idiosyncrasy of the space in a car's interior as an interlocutionary setting was appreciated in the form of Semiotica thematic issue 191, where aspects such as, e.g., talk and activity inside cars have been covered while examining the interior of a car "as socially rich and meaningful" (Haddington et al. 2012: 101). 
With further regard to the semiotics of 'spatiality' of vehicles, Danesi (2008) points out that the "automobile is experienced by many of us as an extension of bodily armor, so to speak. In the public world of traffic, it creates a space around a physical body that is as inviolable as the body itself" (Danesi (2008[1999]: 62). ${ }^{2}$ Building on this claim it could be observed that in phenomenological terms, the space within the confines of the chassis is peculiar in more than one sense. It is still a public sphere in the sense that the people inside are clearly visible to other participants in urban traffic and are liable for any behaviour therein. They have to obey all the binding codes (overt and covert) in a particular community, unlike in a typical private space (a house) where, to a certain extent, an inhabitant is shut off from the outside world. On the other hand, there is also a private axis along which the behaviour of a car owner can be mapped and described as a sort of ambulant spatiotemporality. The ways of customizing this spatio-temporality can be treated as a code: visibility to other participants of the urban setting motivates treating one's vehicle as a carrier for all sorts of messages. ${ }^{3}$ In this way, vehicle interiors can be considered as what Danesi calls a syntext, i.e. a text which "imparts the illusion of connectivity among what would otherwise be perceived as fragmented random texts by simply synthesizing them in an organized fashion" (Danesi 2002: 70).

Figure 1 shows some semiotic instances of disseminating such ideological priorities. During Euro 2012, held in Poland, houses were hardly ever decorated with any national attributes, people did not wear such attributes outside match venues on a day-to-day basis, but the majority of cars circulating around cities were adorned with all types of gadgets stressing support for the Polish national team during most of the competition time (e.g. flags, small toys, towels hung in all possible places in the interior and on the exterior of a car).

Baudrillard turns our attention to the difference, within a hypothetical science of structural technology, between massive technological products such as aeronautics or shipbuilding - where technical pressures maximize structural constraints - and the requirements that car producers are faced with regarding the necessity continuously to exploit every conceivable variation while addressing few simply technological constraints (Baudrillard 2005[1968]: 5). A perfect illustration of this tendency (where the form is in fact counter-productive to function) is Baudrillard's comment on the design of North American cars from the 1950s, which had massive tail fins. As the scholar points out, through this

\footnotetext{
${ }^{2}$ As Danesi further on observes, this perception is not restricted to our culture. He cites the anthropologist Basso who found out, for example, that "the Western Apache of central Arizona also perceive the car as [a] body, even going as far as to use the names of body parts to refer to analogous automobile parts" (Danesi (2008[1999]: 62). ${ }^{3}$ Of course, disregarding purely commercial messages.
} 
formal solution, we "witnessed a veritable triumphalism on the part of the object: the car's fins became the sign of victory over space - and they were purely a sign, because they bore no direct relationship to that victory" (2005[1968]: 62).
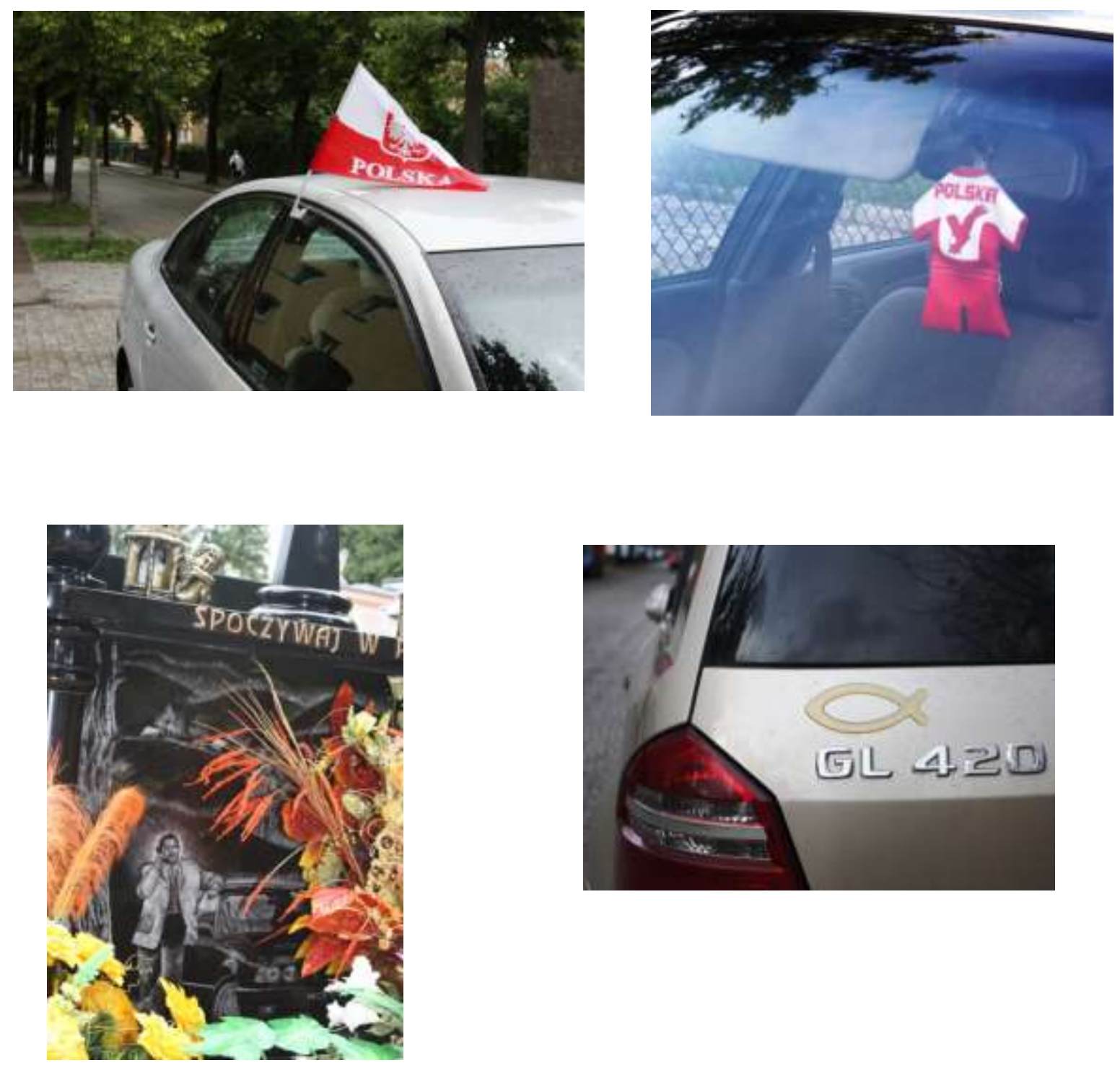

Fig. 1. Cars as carriers of ideological messages. Upper panel: Some of the accessories professing support for the Polish national football team during Euro 2012. The photos taken in a place that did host not any of the matches. Source: MHG. Lower panel: Some of the ways to express transcendental meanings. Left: a photo of a tombstone taken at a Catholic cemetery in Wrocław. Right: the sign of a fish, frequently placed on private cars in Poland.

There was no relationship in that victory because, as Baudrillard further observes, those fins were in fact counterproductive in terms of drag coefficient ${ }^{4}$ and the real velocity that could be attained. The fins were thus representative of a fantasy of aerodynamics (from

\footnotetext{
${ }^{4} \mathrm{Cd}$ (drag coefficient) - a measure of a car's wind resistance.
} 
aeroplanes) as a quasi-enhancement of the Cd value: "Tail fins were a sign not of real speed but of a sublime, measureless speed. They suggested a miraculous automatism, a sort of grace. It was the presence of these fins that in our imagination propelled the car, which, thanks to them, seemed to fly along of its own accord" (Baudrillard 2005[1968]: 63). This type of object thus connotes a technical object in a natural and allegoric way. In automobiles, thus, "the personalization function is not just an added value - it is also a parasitic value. Indeed, from the technological standpoint it is impossible to conceive of an object in an industrial system being personalized without thereby losing some of its technological optimality" (Baurdillard 2005[1968]: 153).

Car design as such has also been studied extensively for its semiotic impact. Karjalainen (2007) points out that apart from its informative function, design serves as a carrier of various symbolic meanings. He suggests that brand design cues are 'value-based' in order to foster solid and consistent recognition, giving the example of BMW using strong shapes and dynamic forms in its cars which clearly communicate BMW's values of performance and power. In particular, he concentrates on the difference between explicit and implicit design cues to create recognition for the brand and discusses the results of projects conducted by his students. He suggests putting a future analytical focus on the coherence between semantic transformation and semantic attribution, as well as on that between design intent and user perception.

Mick et al. (2002), providing an in-depth semiotic overview of consumerism, also mention some landmark elaborations on the semiotics of vehicles. They include Hoshino's (1987) study of commutative and denotative meaning, giving as an example the Tall Boy car (developed by Honda in the early 1980s). However, as Mick et al. observe, the interpretation provided by the author is purely subjective and it is not clear if other researchers would similarly differentiate the connotations, let alone potential consumers (Mick et al. 2002: 10). Odile Solomon, drawing on Jakobson's (actually Brugmann's) communication model, argues that automobile designs have two main communicative functions: phatic and poetic. Blending the characteristics of the phatic function with the Gestalt principles of balance, consistency, grouping and subdivision, she then elaborates on how differentiating the shapes of cars, such as the Volkswagen Beetle (ovoid), Austin Martin (cubic) and Citroen CX (concave trapezoid), influences the memorability of brands. She also extends these insights by revealing the tendencies of meaning in automotive designs across cultures by examining multinational automotive publications and interviews with designers in Japan, America, France, Italy and Germany (Mick et al. 2002: 11). Lefebre's (1989) Barthian analysis deals with the semiotic 
potency of vehicle ownership and care-taking in Pakistan, in particular with decorative paintings on trucks, with cabins and fronts reproducing mosques and Koran quotations, and the sides of trucks showing naturalistic scenery (e.g. mountains, lakes). He concludes that the ornate sign system on Pakistani trucks is meant to show that the driver is a religious and reverent, but courageous, adventurer who owns a gorgeous and prestigious vehicle (Mick et al. 2002: 46). As pointed out above, most of these elaborations mainly show the researcher's voice, their interpretation of design. Our work to check a specified (potential) consumer cohort aimed at improving on this shortcoming.

\section{Semantic differential as an analytical tool}

The SD scale was elaborated and developed by Charles Osgood in a series of publications in the 1950s. In 1946, Stagner and Osgood adapted the idea of 'parallel polarities' to be applied to "the measurement of social attitudes and stereotypes, by using sets of 7-step scales defined by pairs of opposites (e.g. rating PACIFIST against scales like fair-unfair, valuable-worthless, and strong-weak). Later at Illinois (in the early 1950's), this became the Semantic Differential Technique (...). The results clearly demonstrate the universality of three affective features of meaning, Evaluation (E), Potency (P) and Activity (A)" (Osgood 1981: 56f). These three features, known also as the primary dimensions of connotative meaning, "kept reappearing despite deliberate and independent variations in the sampling of scales, of concepts" (Osgood 1971: 171) as three dominant and orthogonal (independent) factors. They had been arrived at through identifying correlations between the scales and then factor-analyzing those scales. The procedure is explained in detail in Osgood (1971: 171). ${ }^{5}$

\footnotetext{
Imagine a space of some unknown number of dimensions. This will be our hypothetical semantic space, and we can explore it by analogy with the more familiar color space. Like all selfrespecting spaces, this one has an origin, which we define as complete "meaninglessness" (analogous to the neutral grey center of the color space). The meaning of a sign can be conceived as some point in this n-dimensional space, and can thus be represented by a vector from the origin to that point: the length of this vector would index the "degree of meaningfulness" of this sign (like saturation in the color space) and its direction would index the "semantic quality" of this sign (analogous to both hue and brightness in the color space). To talk about "direction" in any space requires that we have some reference coordinates (Osgood 1971: 171f).
}

\footnotetext{
5 "The denotative or referential uses of terms-the way the lexicon carves up the world-appear largely arbitrary and unique to particular languages until the ethnolinguist discovers a framework of semantic components that can be imposed comparably on these phenomena" (Osgood 1971: 171).
} 
The three factors are the three dimensions of semantic space. The work above cited reports studies that were carried out to evaluate the generality of affective meaning systems across language and culture groups. The procedures, designed to order qualifier-types in particular languages, were cast in terms of three criteria: (a) maximum overall frequency of usage (salience); (b) maximum diversity of usage (productivity); and (c) minimum correlation in usage (independence) (Osgood 1971: 177).

Since the present paper will be concerned with attitudes, of key importance is the notion of attitude as such. As Minato (1981: 21) admits, in spite of the plethora of definitions of the concept in contemporary psychology and the social sciences, some consensus can be reached. For example, Thurstone (1974 as cited in Minato 1981: 21) defines attitudes as "the sum total of man's inclinations and feelings, prejudice of bias, preconceived notions, ideas, threats and convictions about a specified topic”. Osgood points out that attitudes are learned and implicit, "Further they are predispositions to respond, but are distinguished from other states of readiness in that they predispose toward an evaluative response" (Osgood 1957: 189). Assuming attitudes to be tendencies of approach or avoidance, Osgood contends that attitudes "can be ascribed to some basic or the bipolar continuum with a neutral or zero reference point, implying that they have both direction and intensity and providing a basis for the quantitative index of attitudes. Or, to use a somewhat different nomenclature, attitudes are implicit processes having reciprocally antagonistic properties and varying in intensity" (Osgood 1957: 189-190). Building on these views, Minato assumes that attitudes are "psychological constructs proposed for explaining that fact that that each person responds consistently to a specific objet or a group of objects in a specified way, especially favorably or unfavorably, positively or negatively" (Minato 1981: 21). Hence, attitude can be said to be "a learned implicit process which is potentially bipolar, varies in intensity, and is part of the internal meditational activity that operates between a stimulus and the individual's more overt evaluative response pattern" (Minato 1981: 22).

Another key issue is the so-called polarization of substantives. According to Osgood, the polarization (or affective intensity) of a concept is indexed by its distance from the origin of the semantic space. It can be calculated "either as an average of the absolute deviations of judgments of individual subjects from the midpoints of scales, or by the algebraic average of the deviations for individual subjects - in which case concepts for which different members of the culture have antagonistic meanings will suffer cancellation in polarization toward zero" (Osgood 1981: 188ff). 
Within the thematic focus of the present study we can mention a study by Hsu et al. (2000), who provide an SD analysis of telephone design. ${ }^{6}$ The aim of the study was to stress the usefulness of quantitative data in the study of the relationship between design elements and user evaluation when formulating design strategies. The researchers pointed to the fact that users' feelings about a product involve a complex cognitive process and many various factors contribute to the perception of a product form (Hsu et al. 2000: 376). Designers and users were asked to apply an SD scale to rate their perceptions toward 24 real telephone samples. Subsequently, several multivariate analyses were performed to analyze the subjects' perceptions. The results fell into a separate perceptual space for both subject samples, suggesting that there are crucial differences between designers and users in product form perception. What is more important, the conceptual models of these two subject groups are made up of different components.

\section{Vernacular versus skeuomorphic aspects of car design}

Our analysis will also rely on the dyad of 'vernacular' vs 'skeuomorphic'. Porphyrios (1997[1983]) assumes that, notwithstanding the superficial associations with rusticity that the concept of 'vernacular' evokes, its basic meaning is different: "The idea of vernacular has nothing to do with stylistics. [...] The essential meaning of vernacular refers to straightforward construction, to the rudimentary building of shelter, an activity that exhibits reason, efficiency, economy, durability and pleasure" (Porphyrios 1997[1983]: 179-80) as cited in Evans and Humphrey 2002: 191). As Evans and Humphrey (2002) further stipulate, “"a vernacular', if that term has any validity at all, must relate architectural processes to a given social and technological context. It is then the practical expression in built form of the habitus of social groups. The vernacular is always ordinary and it may even be ugly (Venturi et al., 2000 [1972]) but it cannot be divorced from the experiences and emotional associations of viable everyday life" (Evans and Humphrey 2002: 191). Skeuomorphs, on the other hand, as Evans and Humphrey (2002) further put it, are artefacts which are meant to evoke the appearance of objects made of other materials. They may involve the transformation of previously functional features into decorative ones: "Skeuomorphic architecture is thus likely to spin away from the vernacular, whether vernacular is understood in the direct sense or in the transcendental form" (Evans and Humphrey 2002: 192). The authors further distinguish

\footnotetext{
${ }^{6}$ The same reference for an overview of research on product semantics.
} 
between the sense of a 'mythic order' and symbolism that may assume many contingent forms: "The skeuomorph cannot be seen to 'stand on its own' (of course, no object in fact does this), but inserts itself into relationality from the beginning by virtue of its pretending to be something else" (Evans and Humphrey 2002: 193). As Evans and Humphrey point out, skeuomorphs involve a citation from the original context, "a cipher of cultures and icons of identity" (Evans and Humphrey 2002: 190).

Our preliminary research hypothesis was that, in the particular milieu under analysis, pickups and SUVs (Sports Utility Vehicles) involve a skeuomorphic dimension, semiotically translatable first of all into augmented vehicle dimensions. Such augmented dimensions in a mid-sized town in industrial surroundings are a-teleological - purely symbolic and completely dissociated from any praxis.

Table 1. The specification of the dimensions of a Toyota pickup and a 15 seater bus (van).

\begin{tabular}{|c|c|c|c|}
\hline & a pickup (Hilux) & a van (Hiace) \\
\hline \multicolumn{4}{|c|}{ Maximum Cargo Volume: } \\
\hline \multicolumn{2}{|c|}{ Exterior Length: } & $4980-$ & $4695 \mathrm{~mm}$ \\
\hline \multicolumn{2}{|c|}{ Exterior Width: } & 1760 & $1695 \mathrm{~mm}$ \\
\hline \multicolumn{2}{|c|}{ Exterior Height: } & $(\mathrm{mm}) 1835$ & $1980 \mathrm{~mm}$ \\
\hline \multicolumn{2}{|c|}{ Wheelbase: } & 3085 & 2985 \\
\hline \multicolumn{2}{|c|}{ Curb Weight: } & 1720 & $1690-1855$ \\
\hline \multirow[t]{2}{*}{ engine } & Max torque $^{7}$ & $\mathrm{Nm} 343$ & $241.2 \mathrm{Nm}$ \\
\hline & Max power & $\mathrm{kW} 126$ & $\mathrm{~kW} 75.3$ \\
\hline
\end{tabular}

Let us first take a look at the exemplary dimensional specifications, respectively, of a pickup and a van taken from one brand. Assuming that there is indeed considerable variety across models, the strategy of comparing varieties within one brand should have the advantage of reducing idiosyncratic variation. For the Toyota brand we get the following specifications, as juxtaposed in Table 1. (a pickup model Toyota Hilux, SR 4x4 Extra-Cab Cab-Chassis Turbo Diesel Manual, with common rail injection system, source:. http://www.toyota.com.au/hilux/specifications/sr-4x4-xtra-cab-cab-chassis-turbo-diesel$\underline{\text { manual) }}$ and a van Hiace (2.5 DSL 15 STR AC DLX, a 15 seater type bus). (http://www.pomtco.com/automotive/toyota_hiace_bus_diesel.php). Also 2.5Ltr Turbo Diesel, 4-Cyl, 16V, DOHC, Common-rail type Direct fuel injection system.

\footnotetext{
${ }^{7}$ The rotational force generated by the engine.
} 
For the Mitsubishi make, the results are more drastic. The length of a Mitsubishi pickup L200 (Triton) is longer than that specified for the above Toyota van at $5,185 \mathrm{~mm}$ (http://www.ehow.com/list_7521924_technical-specifications-mitsubishi-1200.html) and a width of $1,815 \mathrm{~mm}$. The transporter's volumetric size is $2.21 \mathrm{~m}^{2}$, with a charging capacity of $960 \mathrm{~kg}$. The 2,477cc engine produces 175 brake horsepower (bhp), with a torque of 350 Newton metres. To compare, the specs for a Mitsubishi L300 van are much less substantial: length: SBW 4,380mm, LBM 4780mm, width 1690mm. Engine capacity is $2533 \mathrm{cc}$ and max torque $195 \mathrm{Nm}$ (http://www.carshowroom.com.au/newcars/2012/Mitsubishi/Express/M9P12A). On this example, pickups actually by far exceed corresponding vans in terms of both dimensions and technical specifications. Finally, a Mitsubishi SUV (http://www.carshowroom.com.au/newcars/2012/Mitsubishi/ASX/MEV12A) ASX 4D Wagon (all three 2012 models) has engine capacity 1998cc (or 1798cc) with max torque 197 $\mathrm{Nm}$, height 1625, width $1770 \mathrm{~mm}$, payload 595kg, and an 'unassuming' length of $4295 \mathrm{~mm}$.

There is one intriguing observation to be made basing on the data from Table 1. A van designed to seat 15 people actually has smaller dimensions and more diminished technical specifications (half as much power, shorter and a narrower wheelbase) than a pickup. The key thing to take into account is that pickups, in the context investigated, are not bought to accommodate any passengers or to carry anything at all. They are usually driven by a single owner. Sometimes the owner buys an additional gimmick: a boot cover, so that the vehicle pretends it could host two additional passenger seats at the back (thus resembling, in that way, a huge SUV - see specimen 2 in Fig. 2). Of course, there are no doors to access this quasipassenger space, hence the cover in question is but another means to boost the overall symbolic space of the vehicle.

It might also be of interest to check the diachronic development of the target model. Let us compare the specifications of exemplary Toyota SUVs (Land Cruisers) from 1997 and 2011, adding the dimensions of a sedan of the same make, as shown in Table 2. A cursory look at these random specifications for an SUV reveals several regularities. First of all, it can be noticed that, in diachronic terms, there has been an addiction to inflating the already huge dimensions: models from 2011 are slightly larger, wider, higher and much heavier compared to models from 1997. On the other hand, there has been a decrease in the cargo-carrying volume, i.e. of the actual praxis of the SUV, how much it can actually carry. There is a thus a double weight impact: with an increase in the 'net' weight of the car from 4,751 lbs. to 5,765 lbs $(2614.96 \mathrm{~kg})$ but an actual decrease in the amount of volume dedicated to carrying stuff. 
There is thus a double decrease of praxis, after increasing the overall dimensions. The volume of the car has been increased and it is able to carry fewer goods. What, in semiological terms, has been increased, what more can it carry? It was the semantic dimension of this message that we hoped to recover using the SD tool. If our supposition was correct, it would mean that pickups would be placed higher than vans, sedans and even SUVs in terms of the dimension of symbolic prestige. We set out to enquire what is really being carried in these huge always empty boots, with the overall dimensions of the vehicle, as shown, being greater than those of vehicles for carrying 15 people - and our supposition was that it is symbolic prestige that owners carry. In other words, driving an oversized car, with a considerable but useless space at the back, and to which one gets access by making the effort of stepping on a sill, conveys a specific message to an 'ordinary' street user.

Table 2. Specification of the changes to the Toyota Land Cruiser model

\begin{tabular}{|c|c|c|c|}
\hline & 1997 & 2011 & Toyota Avalon (sedan 2011) \\
\hline Maximum Cargo Volume: & 90.9 cu.ft & 82 cu. ft. & 14.4 cubic feet \\
\hline Exterior Length: & $189.9 "$ & $194.9 \mathrm{in} 4950 \mathrm{~mm}$. & $197.6 "$ \\
\hline Exterior Width: & 76.0 & 77.6 in $1971 \mathrm{~mm}$ & $72.8 "$ \\
\hline Exterior Height: & $73.6 "$ & $74.0 \mathrm{in} \mid 1880 \mathrm{~mm}$. & $58.5 "$ \\
\hline Wheelbase: & $112 "$ & $112.2 \mathrm{in} \mid 2850 \mathrm{~mm}$. & $111.0 "$ \\
\hline Curb Weight: & 4,751 lbs. & $5765 \mathrm{lbs} \mid 2614.96 \mathrm{~kg}$ & $3,572 \mathrm{lbs}$. \\
\hline
\end{tabular}

\section{Description of the instrument and the analytical procedure}

According to Barthes' semiological principles, a corpus for semiological study is inevitably ridden with immanence: the view from inside. It can be conceived of as "a finite collection of materials, which is determined in advance by the analyst, with some (inevitable) arbitrariness, and on which he is going to work" (Barthes 1986: 96). While the corpus should be broad enough to assume the reasonable premise that its elements will saturate an entire system of resemblances and differences, at the same time it should be as homogenous as possible: both in substance and in time (Barthes 1986: 97). The main goal of the study was to investigate the semiotic values of car design, through which we tried to throw semiotic light on the way "the 
rationality of objects comes to grips with the irrationality of needs", as Baudrillard (2005: 6) concisely observed.

In accordance with Barthes' stipulations, the corpus for the research was compiled so as to cover the maximum range of saliency types, and at the same time we aimed for maximum homogeneity. The target items, as mentioned in the preceding section, were pickups and SUVs. While their design was definitely determined by their function, as used in a cross-country advantage context, we sampled their raison d'être in another context: in a mid-sized town with a reasonably good quality of streets and no dunes or swamps to cross while getting from one suburb to another. To that end, the elapsed time needed to drive through the locality averaged half an hour. An economic particularity of the locality is that the number of pickups and SUVs has been constantly increasing over recent years, just as in other Polish towns and cities, in defiance of the aggravating economic crisis and mounting complaints about the quality of life in all possible media. The research question was thus trying to find a dimension to explain the obviously huge amount of inconvenience (e.g. difficult to park, increased fuel expenses, difficult to drive along relatively narrow streets), coupled with the lack of immediate necessity to use a SUV or a pickup on an everyday basis in a town with a population of about 120.000 inhabitants. ${ }^{8}$

We were thus faced with two constraints on the corpus formation. One was to take into account a wide spectrum of other makes against which to check the semiotic potential of pickups and SUVs, and the opposing constraint was to make the database homogenous, i.e. to eliminate all possible variables that could influence the perception of stimuli. The first filter was colour. We decided to include in the instrument only a specified range of colours of the make. Since the target items were available in the setting only in grey or black, we decided to include filler material which would involve only these neutral colours. In practice, dark blue and dark green also had to be taken into account, but we did exclude bright colours, such as red or bright green or yellow. ${ }^{9}$ Another parameter was car size. The research target was to investigate cars which are quite spacious. Given the huge variety of makes and types of cars on the market, we had to narrow the study down to eliminate the factor of size as well.

\footnotetext{
With a caveat that perceptions of dimensions vary across cultures and time; cf., for example, the issue of a fullsize car in North America. Assuming a certain degree of analytical abstraction, we concentrated on the Polish endemic in a mid-sized town in the 2010s.

${ }^{9}$ For the importance of colour in commodity perception, see e.g. Evas and Lefley (2002). As the authors point out, physical cues and connotative ones do not always match. For example, in terms of scientific descriptors, violet is a 'fast', high-frequency and high-energy colour, while red can be termed a 'slow' and low-energy one (Enas and Lefley 2002: 92). The authors further point out that, in fact, fast cars' bodies are never violet though they are quite frequently painted red. It could be an interesting socio-semiotic fact to examine why pickups or SUVs are never painted red.
} 


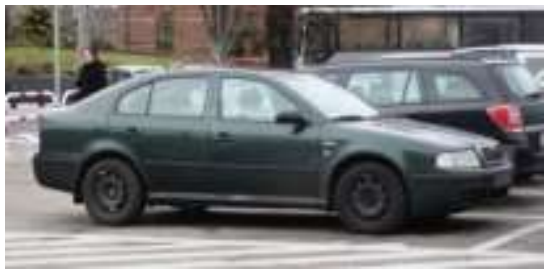

No.1.

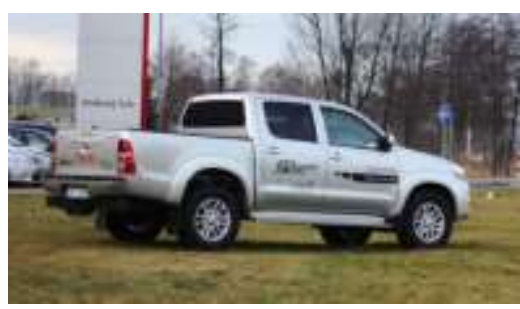

No.4.

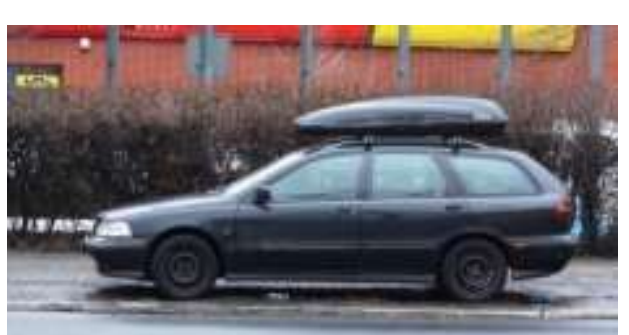

No.7.

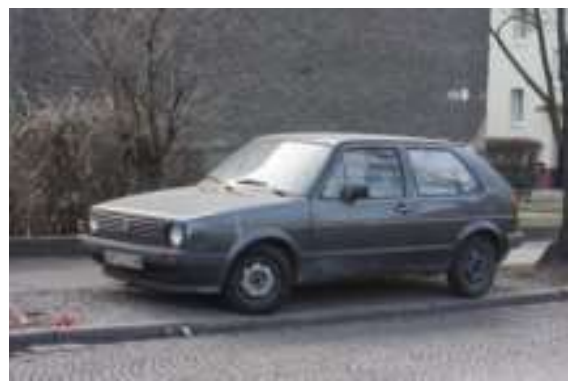

No.10.

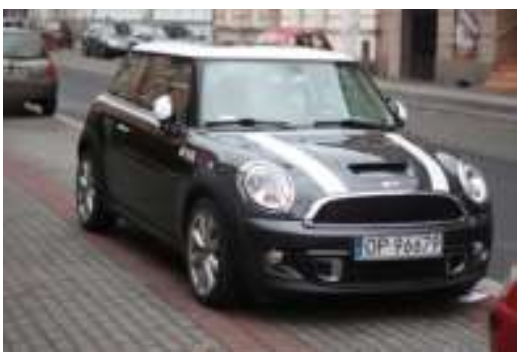

No.13

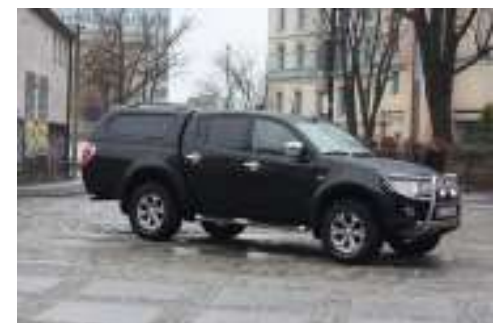

No.2.

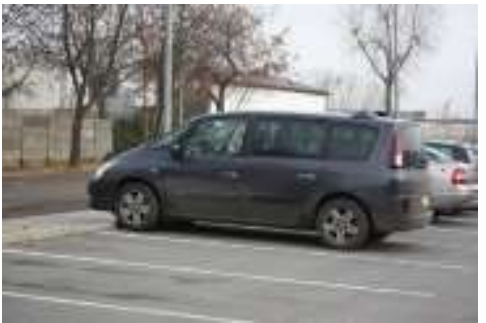

No.5.

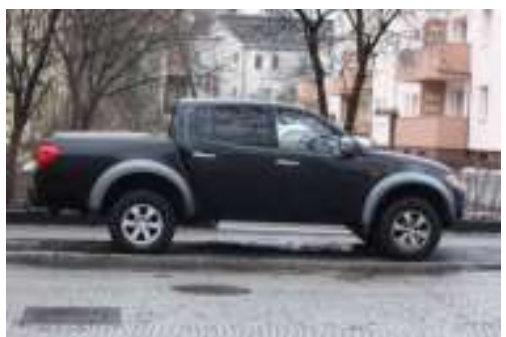

No.8.

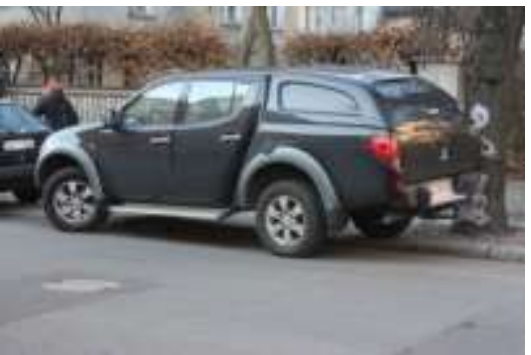

No.11.

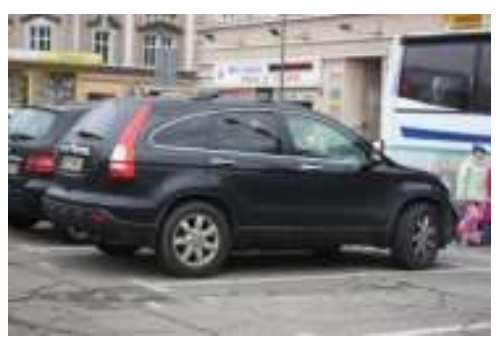

No.14.

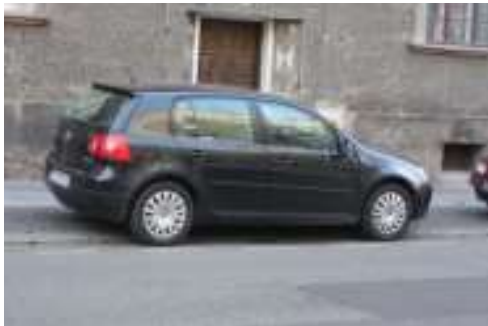

No.3.

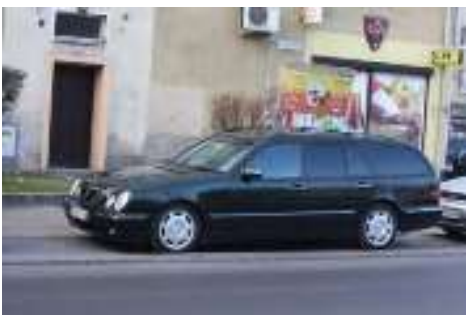

No.6.

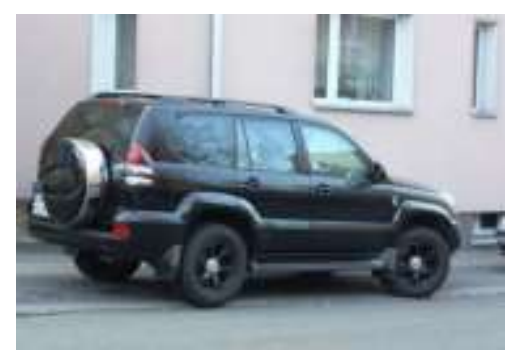

No.9.

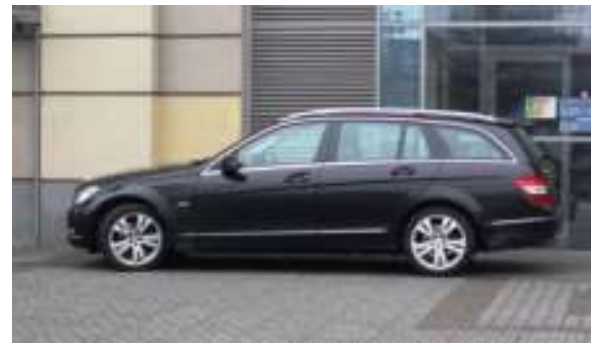

No.12.

Figure 2. Stimuli contained in the instrument. 
That is to say, we included in the instrument only cars that are ipso facto already quite large (or neutral) in terms of dimensions. In practice it meant the exclusion of small cars from the instrument.

It must be pointed out that arriving at a suitable concatenation was very difficult. Several 'interim' versions of the instrument were elaborated. Based on the results of these pilot versions and the feedback from the respondents themselves, the final selection involved the specimens as presented in Figure 2.

[1] was assumed to be a neutral, zero reference point and was discarded in the final calculations. [2, 4, 8, 11] were our first target: pickups, optionally, with and without the boot cover. [9] was a SUV - the second target. Of interest also were [8] and [12] - which were relatively high class, luxury cars. [5] was filler material, with similar parameters to a pickup, but it was a van - a purely functional vehicle. [7] was a common sedan type. The filler material also contained samples of designs which were placed as 'extravagant' outsider, or ideological experiments: a retro-styled Mini Cooper [13] and an old dilapidated Volkswagen Golf [10].

The respondents, both for the pilot study and for the main study, were students at Opole University of Technology (Politechnika Opolska), aged between 21 and 23, with no prior semiotic education background. They were chosen at random from standard departments of a University of Technology, following typical specializations, e.g. Mechanical Engineering, Electrical Engineering, Automatic Control, Civil Engineering, Management, Production Engineering, Logistics, Physiotherapy and Physical Education. The subjects filled in the questionnaire individually in electronic format. A computer with copies of the questionnaire was placed in their classroom during classes and a researcher was present to answer any possible queries. In this way, the respondents did not lose any class time nor did they devote any of their ] free time to the questionnaire. We also wanted to give each person exactly the time they needed to answer fully without pressure or looking to their peers. The instrument was distributed according to these guidelines for several months, starting in February and finishing at the end of the academic year in early June. Altogether we obtained 72 valid answers, but the total number of respondents exceeded this number by a long way: many questionnaires were invalidated due to incorrect completion (e.g. missing replies or double-crossing a scale). The instrument featured two introductory pages, an example page, and 14 pages with stimulus concepts: each stimulus concept was contained on one page beside identical sets of 37 scales. The scaled concepts were as follows (in English translation): 
1. Active-passive

2. emotional - subdued

3. practical --impractical

4. agreeable ----disagreeable

5. dynamic ---- static

6. friendly ---repulsive

7. chaotic-ordery

8. brave ----- covardly

9. selfish ---altruistic

10. unusual -commonplace

11. obtrusive ---discreet

12. strong -weak
13. pleasant -unpleasant

14. congenial (familiar)- strange

15. sociable -unsociable

16. attractive -unattractive

17. noisy --quiet

18. conscientious - unconscientious 30 . intuitive- logical

19. efficient -unefficient

20. progressive - conservative

21. imposing (bossy) - submissive 33. lustful -chaste

22. energetic - lazy

23. fast - slow

24. impulsive - reasonable

36. boastful -modest
25. reliable -unrealiable

26. dangerous -safe

27. successful -unsuccessful

28. kind -unkind

29. rich -poor

31. terryfying -reassuring

32. cheeful - sad

34. hardworking -lazy

35. smoking -nonsmoking

37.competent-incompetent

There are then two possible ways of proceeding: either one can extract a factor structure directly from the data before producing SD scores on the basis of this or one can assume, $a$ priori, that particular scales in the questionnaire belong to Osgood's E, P or A (or some other) dimensions. We chose the first approach, which allows the data to speak for themselves without the pre-imposition of assumptions about the factor structure.

When performing a factor analysis on a data matrix, there are three main things that have to be taken into account:

- the "communalities" of the individual variables (i.e., in our case, the scales);

- the size of the extracted factors ("eigenvalues");

- the size of contribution of each variable to each factor ("loadings").

Communalities are the first stage. If a variable exhibits a low communality, then the model is not working well for that variable and it should be considered for elimination. What counts here as 'low' has been a subject of debate, but MacCallum, Widaman, Zhang and Hong (1999) have suggested that communalities should all be greater than 0.6, and this is what we used. The size and number of retained factors comes next. This has again been widely debated, but a very common criterion (the Kaiser criterion) is to retain only those factors with an eigenvalue greater than 1. A minimum of three variables per factor is also important (Velicer \& Fava, 1998) because a factor with fewer than three items is generally weak and unstable (Costello \& Osborne, 2005). Finally, one needs to consider the loadings. Every scale entered into the factor analysis makes a greater or lesser contribution to each factor (its "loading" on that factor). However, only the highest loading items (either positive or negative) are useful for interpreting the factor and turning it into an SD dimension. Rules of thumb for the optimal minimum value for "high loading" tend to vary in the literature between 
0.4 and 0.6 ; Costello \& Osborne (2005) recommend 0.5 or higher, but values as low as 0.3 have been widely used in practice (cf. Gorsuch, 1983).

With these stipulations in mind, the data were loaded into the program of Hogenraad \& David (1971) and subjected to a between-items principal axis factor analysis with varimax rotation. The first pass of the factor analysis consisted of eliminating the scales with low communalities $(<0.6)$ before re-running it. This led to the retention of just thirteen out of the original thirty-seven scales. The selection that entered final analysis is as follows:

1. Active-passive

2. dynamic ---- static

3. friendly ---repulsive

4. brave ----- covardly

5. obtrusive ---discreet

6. strong-weak
7. pleasant -unpleasant

8. imposing (bossy) - submissive

9. energetic - lazy

10. fast - slow

11. successful -unsuccessful

12. Rich -poor

13. modest-boastful

The next stage of the analysis extracted four factors which met the Kaiser criterion (eigenvalue $>1$ ). However, there was a problem meeting the final criterion of $>3$ items per factor with loadings $\geq 0.6$. As a compromise, the factors 2 and 4 (two items $>0.6$, but everything else $<0.4$ ) were rejected, but factors 1 and 3 (two items > 0.6 but with two more > 0.4 ) were retained. Factors 2 and 4 were pleasantness (F2 = friendly, pleasant) and something to do with excessive size and boastfulness (F4 = obtrusive, boastful). Retained Factor 1 is the potency factor (successful, rich, strong, imposing); retained Factor 3 is the activity/dynamism factor (active, dynamic, fast, brave).

For the SD proper, two dimensions corresponding to Factors 1 and 3 we extracted, using all four of the scales mentioned above for each factor. So, in the subsequent discussion, "Factor 1" is potency and "Factor 2" is activity/dynamism.

To identify the "high" and "low" scoring cars on the SD dimensions, we calculated a 95\% Bayesian credible interval (with a uniform prior) for the mean of the ratings across all cars on each dimension (Curran, 2005). (For a similar procedure, see, e.g., Fleischer, 2003.) A 95\% credible interval is a range of values around a sample statistic and is based on the premise that we want to generalize from the sample to a larger population. A sample statistic - e.g. a sample mean - is usually taken to be a good estimate of the corresponding population parameter, but, owing to sampling error, it may not be an exact estimate. A 95\% credible interval expresses this degree of uncertainty: it gives us a range of values within which we are 95\% certain the true population parameter should lie, even though it may be different from the sample statistic. Observed values which lie above the upper limit of the credible interval can be assumed to exceed the population mean at the $95 \%$ credibility level, and those which lie 
below the lower limit can be assumed to be smaller than the population mean at the same level of credibility.

\section{Results and discussion}

Tartu scholars assumed that

Etant donné que le texte se manifeste dans ces cas par la non-expression, la valeur du message se définit par sa véracité au niveau sémantique de la lingistique globale, et au niveua du 'bon sens'. Pourtant, comme les textes les plus véridiques sont ceux qui ont le plus de credit, il est clair que, là, également, nous avons affair à un sens supplementaire, un sens textual, à côté de la signification linguisitique globale (Lotman - Piatigorskij 1969: 213).

The instrument was devised so as to capture such a supplementary sense evident in the connotative layer of automobile design. Before starting the discussion, we must observe one crucial principle: that of relevance. It basically means keeping to one point of view only, and excluding all that are not associated with this point of view. These other factors, as Barthes (1986: 96) emphasizes, are not denied, they are only ascribed another kind of relevance, "but they must themselves be treated in semiological terms, that is to say that their place and their function in the system of meaning must be determined. Fashion, for instance, evidently has economic and sociological implications; but the semiologist will treat neither the economics nor the sociology of fashion: he will only say at which level of the semantic system of fashion economics and sociology acquire semiological relevance" (Barthes 1986: 96).

An initial hypothesis, as pointed out in the previous section, was to assume a set of pickups as a target group and to posit that they would be placed somewhat higher, for either or both factors, in terms of possessing surplus semiotic value. The remaining material was meant to position and, mathematically speaking, 'to integrate' the target material with selected parameters present in the design of other cars. The key factor was dimensions. In terms of this parameter, pickups share similarity mostly with vans. This is with certain reservations that they are placed closest to vans, although a pickup can far exceed the size of a van. A corresponding SUV model ([8]) does not come near to a pickup in terms of overall dimensions or technical specifications. Hence, the first question was whether there would be any difference between a van and a pickup: a difference will semiotically translate into a connotative impact on purely utilitarian space management. Another factor was the type of engine (assuming that owners, when buying an automobile, are aware of this parameter, although it is not visible to 'the naked eye'): as noted above, pickups have torque exceeding 
that of a van or an SUV. ${ }^{10}$ Let us now discuss the cognitive positioning of the stimuli one by one, with the stimulus concepts (particular cars) referred to heretofore in brackets.

The results of this stage of the analysis are presented in Figure 3. Taking a brief look at the graph seems to imply that the respondents did indeed cognitively group some of the stimuli into semantic clusters, marked on the graph with ellipses. The groupings are generally consistent with semiotic affinity in the design, as discussed above. Of course, these are only suggestions, especially for the medial clusters. However, it can be noticed that all the target items (pickups) are placed relatively close one to another and we can safely posit a cluster status for this stimulus $[2,4,8,11]$. Actually, [11] is placed lowest of all - still, the distance to [8] is visibly much lesser than the corresponding distance to [9] on both axes. There is actually a semiotic explanation for the lowest position of this item, which we will adduce later on.

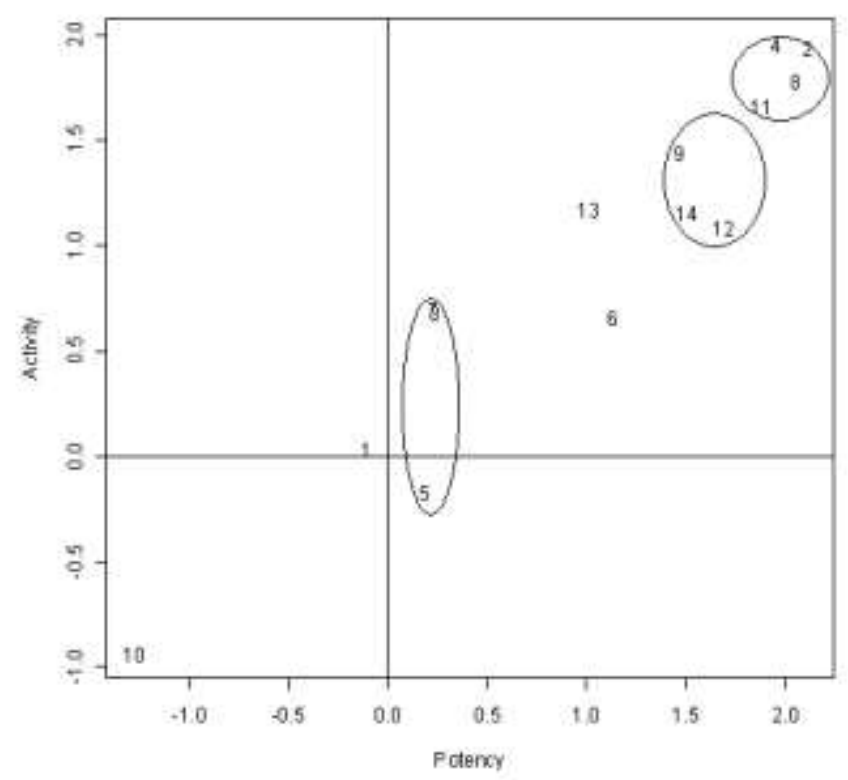

Fig. 3. A semantic space for the two extracted factors.

Let us now discuss the cognitive positioning of the stimuli one by one, starting with the cluster containing the specimens $[7,3,5]$. [7 and 3] ${ }^{11}$ are placed on practically the same level for both factors and share with [5] a similar position on the potency axis. Incidentally, [7 and 5] were the only cars which were theoretically assessed prior to the empirical analysis as

\footnotetext{
${ }^{10}$ Of course, the pickups' torque comes nowhere close to, e.g., 2013 Aston Martin's V12, with a torque of $420 \mathrm{Nm}$ and horsepower of 510. Still, an excess in engine power can be noticed compared with SUVs and vans, taking into account that, by definition, pickups have space for only two people.

${ }^{11}$ At this point we cannot provide any explanation for the patterning of [3] in this group: perhaps the reason was simply that it definitely does not belong to luxury items and the respondents evaluated it as closer to a 'practical' vehicle (i.e. by default not luxury).
} 
having a high criterion of functionality (the vernacular): [5] - a van, and [7] - a sedan type with a car roofbox. [5], a van, has the same dimensions in purely technical terms as the target group of pickups, but these dimensions of pickups in the context of the research performed, as stipulated above, are both skeuomorphic and symbolic. Leaving aside [10], which was placed in the instrument for illustrative purposes mainly, [5] has the lowest rating for activity (dynamism axis) and it contrasts acutely with its skeuomorphic counterpart, the pickups (the group $[2,4,8,11]) .^{12}$

[13], as mentioned before, was included in the instrument as one of the 'odd' examples in the filler material: it shows a small car (a Mini) with retro styling. It terms of the semiotics of design, it does not share any attributes with any of the target or remaining filler material. Since it was placed towards the final part of the instrument, the respondents had already had the time cognitively to construe a semantic space involving the remaining brands. As can be seen, the SD result reflects this atypical status of the vehicle. It can be seen as a harmonic type for our material, placing similarly (high) values for both factors, practically midway between the groups with the highest and lowest ratings. It seems that the respondents in general ranked the owner as relatively dynamic and socially prestigious: in a sense, moderately positive. Another 'oddity' in the filler material was item [10], showing a small automobile of quite shabby appearance. It also was included as additional background to the main research. Placing it as No. 10, towards the end of the instrument, ensured that, similar to the case of [13], it would not influence the responses and yet might provide a new dimension in the analysis of semiotic potential. As can be seen, it received the worst overall rating in the recovered factors: the lowest rating for dynamism and activity.

[1] was a challenge in the instrument make-up. Definitely, we could not have placed any of the target items as the first segment in the instrument because we wanted the respondents to have adjusted their ratings and created a preconceived semantic space before a target model entered the picture. Hence we opted for a model which we thought would be quite neutral: not too shabby or glamorous in appearance and which would be, so to speak,

\footnotetext{
${ }^{12}$ This fact, odd as it may seem, corroborates equally surprising results for the study of vernacular objects reported in Haładewicz-Grzelak -Wilson (2013): in that study, vernacular wayside shrines as opposed to (skeuomorphic) wayside shrines in Licheń (a contemporary Catholic mega-pilgrimage venue in Poland) were subjected to the same type of analysis (SD) and the results confirmed this pattern: although the respondents in that study declared themselves to be 98 per cent Catholics, at the same time they evaluated negatively typical country wayside Catholic shrines, and their highest rating for evaluation was attributed to Lichen sculptures least resembling religious objects or involving many external citations. On the other hand, the parallel is not complete in the sense that we could not recover the evaluation factor at this stage (pleasant, friendly, nice, helpful, etc.), and so, strictly speaking, the issue is evaluation on the activity/ potency axis, and not the evaluation factor as such.
} 
semiotically transparent (relatively large, quite popular within the area the research was conducted). As can be seen, the results reflect this 'tentative' status of the first item: the respondents placed it practically at the neutral (zero) point for both significant factors.

A clear semiotic cluster that emerged at this stage was a group involving [9, 12,14]. This cluster involves all the luxury items from the database - a SUV and a hybrid [14] are included - except the target ones (pickups). In compliance with the initial hypothesis, the luxury items were not grouped with the pickups but formed a visibly separate cluster, collocating slightly lower on both axes than the target items but still visibly higher than the remaining material. Interestingly, a SUV was patterned with a car of a different shape ([12]), which, we hypothetically posited, could semiotically connote more speed (more like a sports car), hence we suspected that perhaps the owner would be rated as more dynamic than the owner of the SUV. Still, the relatively similar position of the two owners in the recovered semantic space would validate the SUV (sports utility vehicle) description.

The cluster $[2,8,4,11]$ was the target cluster - the pickups. The results for this cluster are consistent in a way in that all pickups 'stimuli were placed quite close to each other by the respondents; however, there does not seem to be a difference regarding the pickup version with a skeuomorphic boot cover or without it: items with a boot $([2,11])$ are somewhat 'interspersed' with items without one ([4,8]). To recall, initially we suspected that the version with a boot would be placed higher in the social prestige space than the version without one: in semiotic terms, the boot cover is more skeuomorphic than the boot itself since an uncovered boot could potentially be used to place oversized objects, while the same boot when covered, 'pretending' it is a space to accommodate passengers, gives out a uniform message that nothing ever is carried inside and it definitely cannot be used to transport people since there are no doors to access this skeuomorphic appendix. As can be seen, the results did not corroborate the semiotic impact of the cover as such; nevertheless, the spread of the responses offers two important insights into the semiotics of the accessory in question.

First of all, it is important to note that a pickup with a boot cover was not patterned with the SUVs, which it resembles at first blush, but cognitively was precisely grouped with the pickups. In terms of semiotic potential, the respondents thus 'encrypted' the boot cover as nothing but another skeuomorphic adornment, which does not serve any purpose, just as the empty rail does not serve any purpose in 'town' pickups. So, on the one hand, the message corroborated our hypothesis that the boot cover is skeuomorphic - it is not recognized as functional at all. However, as mentioned above, we also suspected that perhaps the version with the boot cover would be placed higher than versions of pickups without one since, 
theoretically, the volume of skeuomorphic space in an automobile is augmented. While in [4] there is indeed a slight increment along on the activity axis, there is a decrease along the potency axis with respect to an item without a boot cover. And in [11] there is a decrease in both factors. It could thus be posited safely that a boot is indeed perceived as skeuomorphic (the automobile was patterned with pickups, not SUVs) but does not seem to carry additional value in itself.

There is another problem with the results in that, actually, [2] and [11] are practically the same type - though [2] perhaps has more tuning ascribed to it. Yet they were rated relatively differently (possibly assuming polar values within the cluster). In searching for an explanation of the significantly lower position of [11] we were made aware of one issue in the preparation of the instrument. Although we controlled for the colour and dimensions of stimuli concepts, it was impossible to control for graphic presentation, i.e. the exact angle from which the photographs were taken and the surroundings of the automobile, given that the collection of photos took place in a random, streetwise context. [2] is the only photo of a pickup that was taken, so to speak, en face. Additionally, this is a message that was known only to the respondents (residents of the locality in which the photos were taken): the car is parked near the local shopping mall, in a place where no parking is allowed. ${ }^{13}$

In semiotic terms, it might also be noticed that the vehicle in question (a Mitsubishi) has a peculiar design, which makes its front look somewhat shark-like, conveying an impression of aggressiveness. Additionally, it has a set of gadgets (tuning) which, in our paradigm, are to be considered skeuomorphic: pretending as much as possible that the vehicle is to be used off-road in extreme driving conditions (e.g. an additional set of lights, supportive tubing which emphasizes the shark-like lines created by the radiator grill, wing design and headlights). The key is that all this creates a condensed quasi-substance which is 'attached' to the automobile, a substance which would never be used for its real purpose in a mid-sized town in Poland. In the presentation of the stimulus [11], all that 'semiotic substance' is absent precisely because of the way it is shown to the respondents (from the rear). Hence, there is a consistent semiotic explanation for the divergence of perceptions for theoretically the same type of vehicle among our respondents. As can thus be seen, the SD gave us the means to depart from the semiotic level and to dissect particular aspects of design and correlate them with particular axes of factors, reverting back to the semiotic in interpreting factorial results.

\footnotetext{
${ }^{13}$ This seems to be another particularity of a pickup car driver in an urban context. They are usually parked in places where an 'ordinary' citizen would be fined immediately.
} 


\section{Conclusions}

As M. Lotman observes, for a semiotic description there is no principal divide between perceived and not perceived relations and meanings: semiotics allows us to cross the opposition between that received by the senses and that comprehended, because signs explain themselves through signs (M. Lotman 2002: 10).

In this study we have aimed to explore this divide between perceived and not perceived relations as differences in symbolic potential in automobile design by trying to 'flesh out' a semiotic profile of the superfluous in a specific context. We assumed that, judging both by semiotic and technological criteria, there is a surplus value (skeuomorphic dimension) in pickups which we intended to pin down using the tool of SD. The observation was that pickups, a vehicle for a sole driver and no cargo in the context investigated, have the biggest physical dimensions of all personal cars, approximating in fact to a minibus for over ten people. We wanted to enquire how this excess space translated semiotically. We proposed a semiological position to analyze the data, in agreement with Baudrillard's idea that only a semiological model can decipher the meaning structure of a modern commodity, because consumption is defined by the organization of materiality as signifying substance. The results show a specific dimension for superfluous substance in automobile design, rendered as augmented values in the semantic space and with a difference between target items and a 'control' item of the same dimensions (e.g. a van).

This difference in semiotic potential was checked in a specific context and for a specified cohort of young adults. The SD thus gave us the means to dissect particular aspects of the design and to correlate them with particular axes of factors jointly subsumed as social prestige (active/ dynamic and powerful). The results show that surplus semiotic value can indeed be translated directly into positioning in the semiotic space: the owners of pickups were rated highest on both factors and the owners of others luxury cars beneath them, while still maintaining a significant distance from ‘ordinary' vehicles. As far as social competence is concerned (pleasantness, friendliness, helpfulness, etc.), we could not recover significant results at this point. The study should thus be considered a first foray into the semiotic exploration of symbolic potential in car design using the tool of SD; and already, at this stage of the research, the results confirm Baudrillard's definition of consumption as a systematic act involving the manipulation of signs. 


\section{References}

Barthes, Roland, 1986 [1964]. Elements of semiology. Paris: Editions de Seuil.

Baudrillard, Jean. 1997 [1968]. The System of Objects, [trans. James Benedict], London \& New York: Verso.

Baudrillard, Jean. 2005 [1968]. The system of objects. London: Verso.

Curran, James, M. (2005). An introduction to Bayesian credible intervals for sampling error in DNA profiles. Law, Probability and Risk, 4, 115-126.

Danesi, Marcel. 2008[1999]. Of cigarettes, high heels, and other interesting things: An introduction to semiotics. New York: Palgrave Macmillans.

Danesi, Marcel. 2002.Understanding media semiotics. London: Arnold

Evans, Christopher - Caroline Humphrey. (2002). After lives of a Mongolia yurt: The archeology of a Chinese tourist camp. Journal of Material Culture. 7(2). 189- 210.

Eves, Bob - Martin Lefley. 2002. The Color Concept Generator: An adaptive fuzzy color semiotic design tool. Semiotica 142-1/4 91-105.

Genosko, Gary. 1994. Baudrillard and signs: Signification ablaze. London: Rutledge.

Gorsuch, Richard (1983). Factor analysis (2nd ed.). Hillside, NJ: Lawrence Erlbaum Associates.

Fleischer, Michael. 2003. Wirklichkeitskonstruktion: Beitrage zur systemtheoretischen Konstruktivismusforschung. Dresden: Thelem.

Haddington, Pentti - Maurice Neville - Tina Keisanen (2012). Meaning in motion: sharing the car, sharing the drive. Semiotica 191. 101-116.

Haładewicz-Grzelak, Małgorzata - Andrew Wilson. 2013. Mapping skeuomorphic artefacts among Polish young adults: A semantic differential study of sculptures at the Licheń pilgrimage centre. Polish Sociological Review. (181)1. 103-128.

Hogenraad, Robert and Jules, Simon. Structures affective et descriptive dans les jugements d'impressions de l'espace. Psychologica Belgica, 1977, 17, 5-23.

Hoshino, Katsumi (1987). Semiotic marketing and product conceptualization. In UmikerSebeok 1987: 41-56.

Hsu, Shang - Ming C. Chuang - Chien C. Chang. 2000. A semantic differential study of Designers' and users' product form perception. International Journal of Industrial Ergonomics 25 375- 391.

Jakobson, Roman O. 1973. Essais de linguistique générale. Vol. II. Rapports internes et externs du language. Paris: Les Éditions de minuit. 
Karjalainen, Toni-Matti. 2007. It Looks Like a Toyota: Educational Approaches to Designing for Visual Brand Recognition. International Journal of Design, 1(1), 67-81.

Available online at http://www.ijdesign.org/ojs/index.php/IJDesign/article/view/43/14

Lane, Richard. 2000. Jean Baudrillard: Rutledge essential thinkers. London: Taylor and Francis.

Lefebvre, Alain (1989). The decorative truck as a communicative device. Semiotica 75 (3/4), $215-227$.

Lotman, Yurij M. - Alexandre Mosjejevič Pjatigorskij. 1969. Le texte et la fonction. Semiotica 1(2): 205-217.

Lotman, Jurij Mihajlovich (2002). Rol' dyal'nyh modelej v dinamike rysskoj kul'tury. [The role of dual models in the dynamics of Russian culture.] In: Lotman, Jurij Mihajlovich (ed.). Istorija i tipologija russkoj kul'tury. [History and Typology of Russian Culture.] Sankt-Peterburg: Iskusstvo. 88-116.

Lotman, Mihhail. 2002. Semiotika kul'tury v tartusko-moskovskoj semioticheskoj shkole. [Cultural Semiotics in Tartu-Moscow School of Semiotics.[In Russian] In: Lotman, J. (ed). 2002: 5-20.

MacCallum, Robert; Kieth Widaman; Shaobo Zhang and Sehee Hong (1999). Sample size in factor analysis. Psychological Methods, 4(1), 84-99.

MacCallum, Robert; Keith Widaman; Christopher Preacher and Sehee Hong (2001). Sample size in factor analysis: The role of model error. Multivariate Behavioral Research, 36, 611637.

Minato, Saburoh. 1983. Some mathematical attitudinal data on eight grade students in Japan measured by a semantic differential. Educational Studies in mathematics. (14). 19-38.

Mick, David Glen - James E. Burroughs - Patrick Hetzel - Mary Yoko Brannen (2004). Pursuing the meaning of meaning in the commercial world: An international review of marketing and consumer research founded on semiotics. Semiotica 152-1/4 174.

Mortelmans, Dimitri. 2005. Sign values in processes of distinction: The concept of luxury. Semiotica 157-1/4. 497-520.

Osgood, Charles. Semantic Differential Technique in the Comparative Study of Cultures. American Anthropologist, New Series, Vol. 66, No. 3, Part 2. 171-200. 
Osgood, Charles. (1981). The cognitive dynamics of synesthesia and metahphor. Review of Research in Visual Arts Education. Vol. 7, No. 2(14), 56-80.

Porphyrios, Demetri (1997 [1983]). Classicism is not a Style. In: Charles Jencks and Karl

Kropf (eds.) Theories and Manifestoes in Contemporary Architecture. 179-81.

Chichester: Academy Editions.

Poster, Mark. 2001. (ed.) Jean Baudrillard: Selected writings. Stanford: Stanford University

Press.

Poster, Mark. 2001. Introduction. In: Poster (ed.). 1-9.

Solomon, Odile (1988). Semiotics and marketing: New directions in industrial design

Applications. International Journal of Research in Marketing 4 (3), 201-215.

Solomon, Odile (1992). Aesthetic references and cultural representations. In: Objects and

Images, S. Vihma (ed.), 146-158. Helsinki: University of Industrial Arts.

Umiker-Sebeok, Jean (ed.) (1987). Marketing and Semiotics: New Directions in the Study of

Signs for Sale. Berlin: Mouton de Gruyter.

Biographical Note: Andrew Wilson, Ph.D., is a senior lecturer in the Department of Linguistics and English Language in Lancaster University. His main research interests are in quantitative linguistics and computer assisted content analysis. His current research focuses mainly on applying quantitative models to the Celtic languages. He is also interested in non-verbal semiotics, especially the construction of cultural meanings in the domain of fashion and footwear. His recent publications include (2012) "Using Corpora in Depth Psychology: a Trigram-based Analysis of a Corpus of Fetish Fantasies”; (2013) Probability distributions of grapheme frequencies in Irish and Manx: Embracing Bayes factors for key item analysis in corpus linguistics; (2011) "The regressive imagery dictionary: a test of its concurrent validity in English, German, Latin, and Portuguese".

E-mail: a.wilson@lancaster.ac.uk

Biographical Note: Małgorzata Haładewicz-Grzelak (b. 1966) is a senior lecturer in Politechnika Opolska [Opole University of Technology] in the department of Foreign Languages. Her research interests include social semiotics and Beats-and-Binding Phonology. Her recent publications include : (2013) "The analysis of Licheń's Holy Icon as a case study in semiotic fortition" with: Joanna Lubos-Kozieł; (2013) "Ecological aspects of Beats-and-Bindings as exemplified on the comparison of word-medial clusters in English and Spanish" and "The segmentation of phenomenological space in Licheń as an example of double binds" (2014). E-mail: m.haladewicz-grzelak@po.opole.pl 